\title{
How Big Problem Nutrition in Indian Chilren and Adult Population
}

\section{Mishra P*, Mahapatra AK and Moharana S}

IMS and Sum Hospital, SOA University, Bhubaneswar, Odisha

*Corresponding Author: Mishra P, IMS and Sum Hospital, SOA University, Bhubaneswar, Odisha.

Received: November 15, 2019; Published: November 20, 2019

DOI: 10.31080/ASNH.2019.03.0555

\begin{abstract}
Over the years of overweight has becoming an important health index in India. Overall 32 - 33\% of people in India overweight or Obese. It has created huge health and financial problems for a growing economy like India. Today it create problems for. the patient, Society, administration and above all for the Nutritionist. To prevent such an menace, it requires awareness and multiprong approaches. In this review We have highlighted the problems of overweight and obesity and highlight the recommendations which by the Government of India.
\end{abstract}

Keywords: Nutrition; Recommendations

\section{Introduction}

Obesity is defined as abnormal or excessive fat accumulation that presents a risk to health. A crude population measure of obesity is the body mass index (BMI), a person's weight (in kilograms) divided by the square of his or her height (in metres). A person with a BMI of 30 or more is generally considered obese [1].

28 May 2019 - WHO and UNICEF released the "Recommendations for data collection, analysis and reporting on anthropometric indicators in children under 5 years old" guidance document. This document is intended as a reference for the recommended steps in collecting, analyzing and reporting malnutrition estimates based on anthropometric data in nationally representative surveys. Its objective is to set out standardized methods for generating representative malnutrition estimates based on anthropometric data relating to weight, length/height and age in children less than 5 years old. Improving data quality will fill up data gaps and will allow countries to track their progress towards the Global Nutrition Targets for 2025 and the SDGs for 2030 more effectively [1].

Obesity is a complex multifactorial chronic problem developing from interactive influences of numerous factors- social, behavioral, psychological, metabolic, cellular and molecular (genetic). Obesity has been define as a body mass index (BMI) Greater than30 kg/ $\mathrm{m}^{2}$ with extreme define as $40 \mathrm{~kg} / \mathrm{m}^{2}$. It is rapidly becoming leading cause of preventable death in various countries [2].

A new study reported that 102 million men and 101 million women under weight in India leads the world being home to over $40 \%$ of Global underweight. In china $8 \%$ are men and $12 \%$ are women are underweight. The study use data from National and similar studies representing that 19.2 millions adult from 186 countries to arrive its conditions. It is used standard deviation- BMI less than $18.5 \mathrm{~kg} / \mathrm{gm}$ and Obesity $>30 / \mathrm{kg} / \mathrm{gm}$. It is standardized in various age groups $[4,5]$.

In past 10 years, the number of obese people has doubled in the country, according to the National Family Health Survey (NFHS-4). Most of the states have experienced sharp rise in the number of obese people. Andhra Pradesh, Andaman and Nicobar, and Sikkim have more than $30 \%$ of their populations falling under the "obese" category. More than $10 \%$ population in Bihar, Madhya Pradesh, Meghalaya, Tripura and West Bengal. 
Most common facts for obesity and overweight cases

- Consuming excessive calories.

- Leading a sedentary lifestyle without exercise.

- $\quad$ Not sleeping enough: when a person does not sleep enough his body produces ghrelin, a hormone that stimulates appetite. At the same time, a lack of sleep also results in a lower production of leptin a hormone that suppresses the appetite.

- Endocrine disrupters: Scientists believe there is a link between high consumption of fructose and obesity, metabolic syndrome. Animal studies have found that when obesity occurs due to fructose consumption there is a close link with type-2 diabetes [11].

- $\quad$ Age and sex: It can occur at any stage in either sex as long as a person is under positive energy balance. Study conducted at Nutrition Foundation of India shown more females than males are found to be obese among all age groups. Hormonal predisposition put women at higher risk of obesity when compared to men.

- Stress: Food is one of the many stimulants of endorphin, 'feel good' neurotransmitter. Self gratification, self punishment, depression, anxiety and stress may lead to excess caloric intake.

- Endocrine factor: Obesity is found in hypothyroidism, and Cushing's syndrome. Obesity is common at puberty, pregnancy and menopause [2].

- Commercial promoting unhealthy food.

- Consumption of sugar increased.

- $\quad$ Malls, KFC.

- Consumption unhealthy foods and fast food outlet are dramatically changed the lifestyle of family with money to spare.

\section{Consequences}

People who have obesity, compared to those with a normal or healthy weight, are at increased risk for many serious diseases and health conditions, including the following:

\section{High blood pressure (Hypertension)}

The Framingham Heart Study, a famous study for 44 years, estimated that excess body weight (including overweight and obesity), accounted for approximately 26 percent of cases of hypertension in men and 28 percent in women. Individuals with obesity have an increase in fatty tissue that increase their vascular resistance and in turn increases the work of the heart has to do to pump blood throughout the body [14].

\section{Type 2 diabetes}

Havard University researchers found that obesity causes stress in a system of cellular membranes called endoplasmic reticulum (ER), which in turn causes the endoplasmic reticulum to suppress the signals of insulin receptors, which then leads to insulin resistance.

\section{Coronary heart disease}

Due to increased LDL and decreased HDL cholesterol level atherosclerosis (hardening of the heart arteries occurs which causes heart disease [14].

Osteoarthritis a breakdown of cartilage and bone with a joint

Carrying extra weight causes more stress on the joints and tends to accelerate the onset of osteoarthritis.

Some cancers endometrial, breast, colon, kidney, gallbladder

Obese people often have chronic low level inflammation, which can, over time, cause DNA damage that leads to cancer. Chronic local inflammation induced by gastroesophageal reflux disease is a likely cause of esophageal adenocarcinoma. Obesity is a risk factor for gallstones, a condition characterized by chronic gallbladder inflammation, and a history of gallstones is a strong risk factor for gallbladder cancer. If it continue to grow at the same rates the whole world suffering extra cancer causes in the county. Mc Donald's recently step by displaying calorie content of its fast food, but what type of fat or how much of sugar or salt taken as per nutritive value.

Treatment

For children $1 / 2-2$ pounds of weight loss per week and for adults $10 \%$ weight loss in 6 months is recommended. Patient education on lifestyle management is required. Regular 1 to $2 \mathrm{hr}$ physical activity is required. For children one hr of active play, for adolescents aerobic physical activity, muscle-strengthening etc. and for adults at least 150 minutes of moderate intensify exercise $\geq 5$ days/week, at least 75 minutes of vigorous exercise $\geq 3$ days/week are recommended. Appropriate portion sizes of whole grains, 5 or more servings of fruits and vegetables, lean meats and dairy should be included and high calorie foods, drinks including alcohols should be excluded. Proper sleeping timing should be maintained [14]. 


\section{Prevention}

Researchers around the world are trying to find the solutions to the problem of obesity in several ways like providing scientifically based information to the health charities and organizations. to make appositive impact on reducing obesity rates in India.

Obesity is vexing problem in the developed economics. The government should take care to prevent the obesity like: Infrastructure development- Park, pavement; Exercise and nutrition curriculum in schools. Colleges and IT offices. Our society should encourage traditional Indian solutions like: yoga, stress, meditation, and dance. Doctors and clinical dieticians to produce awareness $[14,15]$. Advocacy for change in lifestyle- Assessment of BMI at least annually, and monitoring of increasing BMI should be done. Review should be done of dietary and physical activity habits in addition to sleep duration. Fat free or low fat milk and milk products. If lactose-intolerant, choose fat-free lactose-free milk, or fatfree plain soy, almond, or soya milk. Lean meats, skinless poultry, fish, beans, soy products, eggs, and nuts. Bake, broil, steam, or grill. Avoid frying foods. Low saturated fats (look for $5 \%$ or less on the nutrition facts label). Consider using plant based oils such as olive oil for cooking instead of margarine or butter. To promote exercise in different communities. Use media and classrooms to spread nutrition awareness. Limit TV watching in children. Limit use of mobile. Joint family activities: walks, picnic, sports etc [13-15].

\section{Conclusion}

Obesity has increased by 3 - 4 folds in last decades. It is an uniform problem across the world. Obesity leads to various diseases and result in hospitalization, significant job loss and reduce the longitivity. Over the years, life style and food intake patterns have changed which might have resulted in the specific trends by their complex social-political interactions. Epidemic of obesity needs to be tackled at several levels like: individual level, community level, government level. It needs to be addressed at the socio-economic level as well as in the biological and behavioral areas. By effective prevention of obesity we can hope for a healthy world [16,17].

\section{Bibliography}

1. Recommendations for data collection, analysis and reporting on anthropometric indicators in children under 5 years old: WHO-UNICEF, Updated (2019): 1-144.

2. Ryon T Hurt., et al. "The obesity epidemic challenges, health initiatives and implication for gastroentrities 6.12 (2010): 780-792.
3. Srilakshmi B. "Diet in Obesity and Underweight: Text book of Dietetics: New Age International (P) Ltd (2014): 232-256.

4. Mckay B. "Study Finds Nearly 29\% of the world's Population Is overweight or Obese". The Wall Street Journal 29 (2019).

5. Guthrie Amy. "Number of Overweight in Developing Countries Rises". WHO in Wall Street Journal (2016).

6. National Family Health Survey 2015-16 (NFHS-4) international Institute of population Science (IIPS) updated (2019): 1-6.

7. Pandey K. "India's Obesity Doubled in 10 Years: NFHS-4: a nutritional survey (2016).

8. Bhattacharya S. "India Has a Growing Obesity Problem, Study Shows". The Wall Street Journal (2016).

9. Shannawaz M and Arokiasamy P. "Overweight/obesity: An emerging epidemic in India". Journal of Clinical and Diagnostic Research 12.11 (2018): 1-5.

10. Ahirwar R. "Prevalence of Obesity in India: A Systematic Review". Diabetic and Metabolic Syndrome 13.1 (2019): 318-321.

11. Brazier Y. "What is obesity and what causes it?" 2, Medical News Today (2018).

12. Shimobayashi M. "Insulin resistance causes inflammation in adipose tissue". Junior Chamber International 128.4 (2018): 1538-1550.

13. Pi-Sunyer Xavier F. "Clinical Guidelines on The Ientification, Evaluation, and Treatment of Overweight and Obesity In Adults". Centers of Disease Control and Prevention 15 (): 1-262.

14. Delaney J. "Hypertension and Obesity: How Weight-loss Affects Hypertension". OAC Community 800 (2019): 717-3117.

15. Orringer A Kelly. "Obesity Prevention and Management". Guidelines for Clinical Care Ambulatory University of Michigan 6 (2019): 1-8.

16. Unnikrishnan Ambika., et al. "Preventing Obesity in India: Weighing the options". Indian Journal of Endocrinology and Metabolism 16.1 (2012): 4-6.

17. Uma $\mathrm{V}$ and Selvaamani Y. "Risk of health among adults in India". NFHS 324-327.

Volume 3 Issue 12 December 2019

(C) All rights are reserved by Mishra $P_{.}$, et al. 